\title{
Bayesian analysis for cystic fibrosis risks in prenatal and carrier screening
}

Shuji Ogino, $M D, P h D^{1-3}$, Robert B. Wilson, $M D$, $P h D^{4}$, Bert Gold, $P h D^{5}$, Pamela Hawley, $M S^{6}$, and Wayne W. Grody, MD, $P h D^{7}$

\begin{abstract}
Purpose: Risk assessment is an essential component of genetic counseling and testing, and Bayesian analysis plays a central role in complex risk calculations. We previously developed generalizable Bayesian methods to calculate the autosomal recessive disease risk of a fetus when one or no mutation is detected, and another, independent risk factor is present. Our methods are particularly useful for calculating the CF disease risk for a fetus with echogenic bowel. In genetics practice, however, there are other scenarios for which our previous methods are inadequate. Methods and Results: We provide herein methods for calculating genetic risks in a variety of common clinical scenarios. These scenarios include the following: (1) different mutation panels that have been used for the parents and for a fetus; (2) genetic testing results available on the proband or other relatives, in addition to the consultand; (3) fetal ultrasound negative for echogenic bowel with a positive family history; and (4) a consultand with a mixed ethnic background. Conclusion: Our Bayesian methods have proven their versatility through application to many different common genetic counseling scenarios. These methods permit autosomal recessive disease and carrier probabilities to be calculated accurately, taking into account all relevant information. Our methods allow accurate genetic risk estimates for patients and their family members for CF or other autosomal recessive disorders. Genet Med 2004:6(5):439-449.
\end{abstract}

Key Words: CFTR, counseling, cystic fibrosis, probability, screening

Risk assessment is an essential component of genetic counseling and testing, and Bayesian analysis plays a central role in complex risk calculations. ${ }^{1-4}$ We previously developed generalizable Bayesian methods to calculate the risk of an autosomal recessive disease when only one or no mutation is detected in the disease gene, and another, independent risk factor is present [e.g., fetal echogenic bowel as a risk factor for cystic fibrosis (CF; OMIM no. 219700)]. ${ }^{5}$ Our methods are useful in the setting of $\mathrm{CF}$ carrier screening and prenatal testing for mutations of the CFTR gene (OMIM no. 602421). ${ }^{5}$ In genetics practice, however, there are other scenarios for which our previous methods are inadequate. Such scenarios are common, and generalizable methods for them are lacking. In this manuscript, we illustrate principles of genetic risk calculations for a

\footnotetext{
From the ${ }^{1}$ Department of Pathology, Brigham and Women's Hospital; ${ }^{2}$ Department of Medical Oncology, Dana-Farber Cancer Institute; ${ }^{3}$ Harvard Medical School, Boston, Massachusetts; ${ }^{4}$ Department of Pathology and Laboratory Medicine, University of Pennsylvania Medical Center, Philadelphia, Pennsylvania; ${ }^{5}$ Human Genetics Section, Laboratory of Genomic Diversity, National Cancer Institute at Frederick, Frederick, MD; ${ }^{6}$ Department of Medicine, The Children's Hospital, Boston, Massachusetts; and the ${ }^{7}$ Departments of Pathology and Laboratory Medicine, Human Genetics, and Pediatrics, UCLA School of Medicine, Los Angeles, California.

Shuji Ogino, MD, PhD, Department of Pathology, Brigham and Women's Hospital, Harvard Medical School, 75 Francis St., Boston, MA 02115.E-mail: shuji_ogino@dfci.harvard.edu Received: May 17, 2004.

Accepted: June 28, 2004.
}

DOI: 10.1097/01.GIM.0000139511.83336.8F variety of such scenarios using concrete examples. For example, as the technology of mutation detection advances, more mutations will be screened at lower costs, and mutation panels such as those for CF will likely include many more mutations. Even now, some organizations and institutions provide more mutation coverage than others in a given ethnicity. We provide methods for genetic risk calculations for scenarios in which the proband or another family member has been tested using a different mutation panel from that used for the consultand, and in which the fetus has been tested using a different mutation panel from that used for the parents.

Another common scenario requiring methods beyond those described in our previous report ${ }^{5}$ is that of a pregnant woman with a negative ultrasound who does not undergo prenatal genetic testing. A typical case is as follows: a mother undergoes $\mathrm{CF}$ carrier screening and is found to be a carrier; the father tests negative for CFTR mutations, and the fetus subsequently undergoes ultrasound examination and is found to be negative for echogenic bowel. If the fetus were positive for echogenic bowel, testing for CFTR mutations would usually follow, and genetic risk can be calculated as we described previously. ${ }^{5}$ The fraction of fetuses with a positive ultrasound examination, however, is small, even when two CFTR mutations are present. The probability that a fetus has an echogenic bowel if the fetus is affected with CF, is a carrier, or is a noncarrier is estimated to be $\approx 0.11,0.00089$, or 0.00035 , respectively (using the data by Scotet et al. $\left.{ }^{6}\right){ }^{5}$ Therefore, the probability that a fetus does not 
have echogenic bowel if the fetus is affected, a carrier, or a noncarrier is estimated to be $0.89(=1-0.11), \approx 1(=1-$ $0.00089)$, or $\approx 1$ (=1-0.00035), respectively. This means that a vast majority of pregnant CF carriers who undergo ultrasound examination will have negative results. Women with negative fetal ultrasound results do not often undergo prenatal testing, and accurate genetic risk calculations are needed in this setting. We describe herein Bayesian analyses for various typical scenarios with a negative or positive prenatal ultrasound result, with or without prenatal genetic testing.

In addition, we describe methods for genetic risk calculations in typical scenarios in which a genetic testing result is available in family members other than the consultand or the fetus, and in scenarios in which a consultand with a mixed ethnic background undergoes CF carrier screening. The latter examples are particularly important for countries or geographic regions with ethnic diversity.

Our Bayesian methods are versatile. They are applicable to many common clinical scenarios and allow autosomal recessive disease probabilities and carrier probabilities to be calculated accurately, taking into account all relevant information.

\section{METHODS AND RESULTS}

Table 1 summarizes commonly encountered scenarios that are described in Scenario 1 through Scenario 4. Although these scenarios are relatively common, our methods are applicable to many different, even rare, scenarios. We also describe a variety of other scenarios that pose particularly difficult problems in genetic counseling. We assume that both CFTR testing for carrier screening and CFTR testing for prenatal testing detects the 25 CFTR mutations designated by the American College of Medical Genetics (ACMG). ${ }^{7,8}$ We use mutation detection rates for major ethnic groups as reviewed in Ogino et al. ${ }^{5}$ We use the calculations of Ogino et al. ${ }^{5}$ (from the data of Scotet et al. ${ }^{6}$ ) for the conditional probability that a fetus has an echogenic bowel if affected, a carrier, or a noncarrier $(0.11,0.00089$, or 0.00035 , respectively). We describe methods for risk calculations for more complex scenarios in Scenario 5 through Scenario 9.

\section{Scenario 1: Negative fetal ultrasound, no prenatal CFTR testing}

How can one calculate the genetic risk of CF to a fetus if the mother has a negative ultrasound result and does not

Table 1

Summary of various clinical scenarios of fetal ultrasounds with or without prenatal genetic testing, necessitating genetic risk assessments

\begin{tabular}{lll}
\hline $\begin{array}{l}\text { Fetal } \\
\text { ultrasound }\end{array}$ & Prenatal genetic testing & Scenario \\
\hline Negative & Not performed & Scenario 1 (Tables 2 and 3) \\
$\begin{array}{c}\text { Negative or } \\
\text { Positive }\end{array}$ & $\begin{array}{c}\text { Performed: Negative or one } \\
\text { mutation detected }\end{array}$ & $\begin{array}{c}\text { Scenario 2 and see Ogino et al. } \\
\text { Positive }\end{array}$ \\
Not performed & $\begin{array}{c}\text { Scenarios 3 and } 4 \text { (Tables } 4 \\
\text { through 7) }\end{array}$ \\
\hline
\end{tabular}

undergo prenatal testing for CFTR mutations? First, one calculates the carrier risk for each parent; if the family history is negative for $\mathrm{CF}$ and no carrier screening has been performed, then the carrier risk is the same as that of his or her background population. If there is a family history and/or a test result from carrier screening, then one can calculate the carrier probability accordingly using published mutation frequency estimates and a deduced mutation detection rate for the appropriate ethnic population. $5,7,9$

For example, suppose an Ashkenazi Jewish mother is positive for a CFTR mutation, and the non-Hispanic Caucasian father who does not have a family history of CF is negative for the 25 CFTR mutation panel recommended by ACMG. ${ }^{7,8}$ The fetus undergoes ultrasound examination, and is negative for echogenic bowel. Without prenatal CFTR testing, what is the probability that the fetus is affected (or a carrier)? The Bayesian analysis for this case is shown in Table 2 . The conditional probabilities are derived as follows: For Ashkenazi Jewish and non-Hispanic Caucasian populations, the mutation detection rates of the 25-CFTR-mutation panel are 0.97 and 0.9 , respectively. Therefore, the probability that an Ashkenazi Jewish or non-Hispanic Caucasian parent tests negative if he/she is a carrier is 0.03 or $0.1(=1-$ [mutation detection rate]), respectively. The probability that a parent tests negative if he/she is a noncarrier is 1 , regardless of ethnicity. The probability that the fetus is affected, a carrier or a noncarrier, if the father is a carrier, is $0.25,0.5$, or 0.25 , respectively (because the mother is a carrier). The probability that the fetus is a carrier or a noncarrier, if the father is a noncarrier, is 0.5 , or 0.5 , respectively. The probability that the fetus does not have an echogenic bowel if the fetus is affected, or if the fetus is unaffected (a carrier or noncarrier), is 0.89 , or $\approx 1$, respectively (see earlier). Each column represents a particular combination of conditions. For example, column A represents the combined condition that the father is a carrier, the father tests negative for mutations in CFTR, the fetus is affected, and the fetus is negative for an echogenic bowel. The posterior probability that the fetus is affected is the posterior probability of column $\mathrm{A}$, or $0.000923(\approx 1 / 1100)$, which is approximately 2.3-fold higher than that of a fetus in the general Caucasian population.

If both parents are carriers and their fetus is negative for an echogenic bowel, what is the probability that the fetus is affected? The prior probability that the fetus is affected, a carrier, or a noncarrier before the ultrasound examination is $0.25,0.5$, or 0.25 , respectively. The Bayesian analysis for this scenario is shown in Table 3 . The probability that the fetus is affected is $\approx 0.23$, which is sufficiently high to recommend prenatal genetic testing (as in Scenario 2).

\section{Scenario 2: Negative fetal ultrasound, with prenatal CFTR testing}

If a pregnant woman with a negative ultrasound result undergoes prenatal CFTR genetic testing, Bayesian analysis 
Table 2

Bayesian analysis for a fetus negative for echogenic bowel, with a carrier mother (Scenario 1)

\begin{tabular}{lcccc}
\hline Father & & Carrier & & Noncarrier \\
\hline Prior probability & & 0.04 & 0.96 \\
Conditional probability of negative carrier screening & & 0.10 & 1 \\
Fetus (given carrier mother) & Affected & Carrier & Noncarrier & Carrier \\
& 0.25 & 0.5 & 0.25 & 0.5 \\
Conditional probability of negative fetal ultrasound & 0.89 & 1 & 1 & 0.5 \\
Joint probability & 0.00089 & 0.002 & 0.001 & 0.48 \\
Posterior probability & 0.00092 & 0.0021 & 0.0010 & 0.4980 \\
Column & A & B & C & D \\
\hline
\end{tabular}

Table 3

Bayesian analysis for a fetus negative for echogenic bowel, with carrier parents (Scenario 1)

\begin{tabular}{lccc}
\hline Fetus & Affected & Carrier & Noncarrier \\
\hline Prior probability & 0.25 & 0.5 & 0.25 \\
$\begin{array}{l}\text { Conditional probability of negative } \\
\quad \text { fetal ultrasound }\end{array}$ & 0.89 & 1 & 1 \\
$\begin{array}{l}\text { Joint probability } \\
\text { Posterior probability }\end{array}$ & 0.2225 & 0.5 & 0.25 \\
\hline
\end{tabular}

can be performed as described previously, ${ }^{5}$ with little modification. One should use the probability that the fetus does not have echogenic bowel if the fetus is affected, a carrier, or a noncarrier $\left(0.89, \approx 1\right.$, or $\approx 1$, respectively).$^{5}$

\section{Scenario 3: Positive fetal ultrasound, one carrier parent, the other parent not tested or negative by carrier testing, no prenatal CFTR testing}

If a pregnant woman with a positive fetal ultrasound result is found to have one CFTR mutation, and no genetic testing has been performed on the other parent (a nonHispanic Caucasian) or the fetus, what is the probability that the fetus is affected? The Bayesian analysis for this case is shown in Table 4 . The probability that the fetus is affected is $\approx 0.64$, and prenatal genetic testing (see Scenario 2 ) is indicated. Suppose the father tests negative for mutations in CFTR? The Bayesian analysis for this case is shown in Table 5 . The probability that the fetus is affected is $\approx 0.16$, which is sufficiently high to recommend prenatal genetic testing (see Scenario 2). If one or both of the parents have a family history of CF, and one or more of the other family members have been tested, the risk calculations can become quite complex (see Scenario 5).

\section{Scenario 4: Positive fetal ultrasound, one or both of parents negative by carrier testing, no prenatal CFTR testing}

If one of the parents tests negative for mutations in CFTR, but the fetus has an echogenic bowel, what is the probability that the fetus is affected? Suppose that two non-Hispanic Caucasian parents have negative family histories for CF: Parent A tests negative for mutations in CFTR, and Parent $\mathrm{B}$ has not been tested. The Bayesian analysis for this case is shown in Table 6 . The posterior probability that the fetus is affected is $0.012(\approx 1 / 83)$.

If both parents test negative for mutations in CFTR, but the fetus has an echogenic bowel, what is the probability that the fetus is affected? The posterior probability in this relatively common scenario is important, as it determines whether prenatal CFTR testing should be offered. Suppose both parents have a negative family history for $\mathrm{CF}$, and both

Table 4

Bayesian analysis for a fetus positive for echogenic bowel, with a carrier mother (Scenario 3)

\begin{tabular}{lcccc}
\hline Father & & Carrier & & Noncarrier \\
\hline Prior probability & & 0.04 & 0.96 \\
Fetus (given carrier mother) & Affected & Carrier & Noncarrier & Carrier \\
& 0.25 & 0.5 & 0.25 & 0.5 \\
Conditional probability of positive fetal ultrasound & 0.11 & 0.00089 & 0.00035 & 0.00089 \\
Joint probability & 0.0011 & 0.0000178 & 0.0000035 & 0.0004272 \\
Posterior probability & 0.64 & 0.010 & 0.0021 & 0.00035 \\
\end{tabular}


Table 5

Bayesian analysis for a fetus positive for echogenic bowel, with a carrier mother and the father tested negative (Scenario 3)

\begin{tabular}{|c|c|c|c|c|c|}
\hline Father & \multicolumn{3}{|c|}{ Carrier } & \multicolumn{2}{|c|}{ Noncarrier } \\
\hline Prior probability & \multicolumn{3}{|c|}{0.04} & \multicolumn{2}{|c|}{0.96} \\
\hline Conditional probability of negative carrier screening & \multicolumn{3}{|c|}{0.10} & \multicolumn{2}{|c|}{1} \\
\hline \multirow[t]{2}{*}{ Fetus (given carrier mother) } & Affected & Carrier & Noncarrier & Carrier & Noncarrier \\
\hline & 0.25 & 0.5 & 0.25 & 0.5 & 0.5 \\
\hline Conditional probability of positive fetal ultrasound & 0.11 & 0.00089 & 0.00035 & 0.00089 & 0.00035 \\
\hline Joint probability & 0.00011 & 0.00000178 & 0.00000035 & 0.0004272 & 0.000168 \\
\hline Posterior probability & 0.16 & 0.0025 & 0.00049 & 0.60 & 0.24 \\
\hline
\end{tabular}

Table 6

Bayesian analysis for a fetus with echogenic bowel ${ }^{a}$ (Scenario 4)

\begin{tabular}{|c|c|c|c|c|c|c|c|c|}
\hline Parent A & \multicolumn{5}{|c|}{ Carrier } & \multicolumn{3}{|c|}{ Noncarrier } \\
\hline Prior probability & \multicolumn{5}{|c|}{0.04} & \multicolumn{3}{|c|}{0.96} \\
\hline $\begin{array}{l}\text { Conditional probability of negative } \\
\text { carrier screening }\end{array}$ & \multicolumn{5}{|c|}{0.1} & \multicolumn{3}{|c|}{1} \\
\hline \multirow[t]{2}{*}{ Parent B } & \multicolumn{3}{|c|}{ Carrier } & \multicolumn{2}{|c|}{ Noncarrier } & \multicolumn{2}{|c|}{ Carrier } & Noncarrier \\
\hline & \multicolumn{3}{|c|}{0.04} & \multicolumn{2}{|c|}{0.96} & \multicolumn{2}{|c|}{0.04} & 0.96 \\
\hline \multirow[t]{2}{*}{ Fetus } & Affected & Carrier & Noncarrier & Carrier & Noncarrier & Carrier & Noncarrier & Noncarrier \\
\hline & 0.25 & 0.5 & 0.25 & 0.5 & 0.5 & 0.5 & 0.5 & 1 \\
\hline $\begin{array}{l}\text { Conditional probability of positive } \\
\text { fetal ultrasound }\end{array}$ & 0.11 & 0.00089 & 0.00035 & 0.00089 & 0.00035 & 0.00089 & 0.00035 & 0.00035 \\
\hline Joint probability & $4.4 \times 10^{-6}$ & $7.1 \times 10^{-8}$ & $1.4 \times 10^{-8}$ & $1.7 \times 10^{-6}$ & $6.7 \times 10^{-7}$ & $1.7 \times 10^{-5}$ & $6.7 \times 10^{-6}$ & $3.2 \times 10^{-4}$ \\
\hline Posterior probability & 0.012 & 0.00020 & 0.000040 & 0.0048 & 0.0019 & 0.048 & 0.019 & 0.91 \\
\hline
\end{tabular}

${ }^{a}$ One parent (non-Hispanic Caucasian Parent A) tested negative by carrier screening, but no genetic testing has been performed on the other parent (non-Hispanic Caucasian Parent B) or the fetus.

Table 7

Bayesian analysis for a fetus with echogenic bowel ${ }^{a}$ (Scenario 4)

\begin{tabular}{|c|c|c|c|c|c|c|c|c|}
\hline Parent A & \multicolumn{5}{|c|}{ Carrier } & \multicolumn{3}{|c|}{ Noncarrier } \\
\hline Prior probability & \multicolumn{5}{|c|}{0.04} & \multicolumn{3}{|c|}{0.96} \\
\hline $\begin{array}{l}\text { Conditional probability of } \\
\text { negative carrier screening }\end{array}$ & \multicolumn{5}{|c|}{0.1} & \multicolumn{3}{|c|}{1} \\
\hline \multirow[t]{2}{*}{ Parent B } & \multicolumn{3}{|c|}{ Carrier } & \multicolumn{2}{|c|}{ Noncarrier } & \multicolumn{2}{|c|}{ Carrier } & Noncarrier \\
\hline & \multicolumn{3}{|c|}{0.04} & \multicolumn{2}{|c|}{0.96} & \multicolumn{2}{|c|}{0.04} & 0.96 \\
\hline $\begin{array}{l}\text { Conditional probability of } \\
\text { negative carrier screening }\end{array}$ & \multicolumn{3}{|c|}{0.1} & \multicolumn{2}{|c|}{1} & \multicolumn{2}{|c|}{0.1} & 1 \\
\hline \multirow[t]{2}{*}{ Fetus } & Affected & Carrier & Noncarrier & Carrier & Noncarrier & Carrier & Noncarrier & Noncarrier \\
\hline & 0.25 & 0.5 & 0.25 & 0.5 & 0.5 & 0.5 & 0.5 & 1 \\
\hline $\begin{array}{l}\text { Conditional probability of positive } \\
\text { fetal ultrasound }\end{array}$ & 0.11 & 0.00089 & 0.00035 & 0.00089 & 0.00035 & 0.00089 & 0.00035 & 0.00035 \\
\hline Joint probability & $4.4 \times 10^{-7}$ & $7.1 \times 10^{-9}$ & $1.4 \times 10^{-9}$ & $1.7 \times 10^{-6}$ & $6.7 \times 10^{-7}$ & $1.7 \times 10^{-6}$ & $6.7 \times 10^{-7}$ & $3.2 \times 10^{-4}$ \\
\hline Posterior probability & 0.0013 & 0.000022 & 0.0000043 & 0.0052 & 0.0020 & 0.0052 & 0.0020 & 0.984 \\
\hline
\end{tabular}

${ }^{a}$ Both parents (non-Hispanic Caucasian Parent A and Parent B) tested negative by carrier screening, but no genetic testing has been performed on the fetus.

parents are non-Hispanic Caucasian. The Bayesian analysis for this case is shown in Table 7. The posterior probability that the fetus is affected is $0.0013(\approx 1 / 740)$. If one or both of the parents have a family history of $\mathrm{CF}$, and if one or more family members have been tested, the risk calculations can become quite complex (see Scenario 5). 


\section{Scenario 5: Complex scenarios: Consultand negative by carrier testing, proband with one or two detectable mutations}

If a pregnant non-Hispanic Caucasian woman with an affected nephew tests negative for mutations in CFTR, and the affected nephew has only one detectable mutation, what is the probability that the woman is a carrier? The probability that the woman is a carrier, before carrier screening, is $1 / 2$. The Bayesian analysis is shown in Table 8, where the detectable mutation and the undetectable mutation in the nephew are designated as Mutation A and Mutation B, respectively. The posterior probability that the woman is a carrier is $1 / 3$, which is only slightly decreased from the $1 / 2$ prior risk. Without further testing, the probability that the fetus is affected is $1 / 3 \times 1 / 25 \times 1 / 4=1 / 300$. If the fetus is found to have one detectable mutation, the probability that the fetus is affected is $1 / 3 \times 1 / 2=1 / 6$. This is because the risk that the woman is a carrier derives from the risk of carrying an undetectable mutation; for the fetus to be affected, the mutation must come from the father, and the undetectable mutation must have come from the mother. Suppose the fetus has no detectable mutation and the father is also a non-Hispanic Caucasian. The Bayesian analysis for this case is shown in Table 9. The probability that the fetus is affected is $1 / 2946$, which is approximately $1 / 10$ the risk in the case with no prenatal testing on the fetus.

If the affected nephew has two detectable mutations, the consultand's negative test result decreases her carrier risk to less than that of an individual with a negative test result, the same population background, and no family history (assuming the proband and consultand were tested by the same mutation panel). The remaining risk derives from the possibility of an undetectable mutation in the family, unrelated to either of the detectable mutations in the proband. The source of the undetectable mutation, if any, is from one of the consultand's parents (provided that the other consultand's parent has one of the detectable mutations present in the proband). There is also a small possibility that one of the consultand's parents has two mutations, one of which is undetectable, and one of which causes very mild impairment of CFTR protein function, so that the consultand's parent seems asymptomatic.

\section{Scenario 6: Complex scenarios: Consultand negative by carrier testing, and an unaffected family member with one detectable mutation}

In a modification of Scenario 4 (Table 6), suppose the tested woman has a family history of CF, but the affected nephew has not been tested. Suppose further that an unaffected family member, between the woman and the affected nephew in the pedigree, is found to have one detectable mutation. The woman's carrier risk would be significantly lower than that of an individual who tests negative, and is from the same population background, and whose family history is negative. In general, for a consultand who tests negative and has a family history, a detectable mutation in an unaffected relative lowers the consultand's risk; the remaining risk derives from the possibility of an undetectable mutation in the family, unrelated to the detectable mutation in the carrier family member.

Suppose the tested pregnant woman has a family history of $\mathrm{CF}$, the affected nephew has not been tested, and the mother of the affected nephew, not related to the pregnant woman, is found to have one detectable mutation. The woman's carrier risk would decrease less than in the above case. For example, suppose the woman has an unaffected brother whose son is affected, and this affected nephew has not been tested. The woman tests negative for mutations in CFTR. The mother of the affected nephew is found to have one detectable mutation. If this is a non-Hispanic Caucasian family, what is the probability that the woman is a carrier?

The Bayesian analysis for this case is shown in Table 10. The risk that the woman is a carrier before a mutation is detected in the mother of the affected nephew can be calculated by Bayesian analysis: the prior probability that the woman is a carrier is 0.5 , and the conditional probability of a negative test result if the woman is a carrier or noncarrier is 0.1 or 1 , respectively. The posterior probability that the woman is a carrier is $1 / 11$ (calculation not shown). The prior probability that the woman is a carrier derives from the two mutations present in the affected individual, with equal probability. The affected nephew inherited the detectable mutation (Mutation A) from his mother; therefore, the risk that the woman is a carrier now derives from the probability that the woman carries a mutation other than Mutation A (i.e., Mutation B, which can be identical-by-state with Mutation A). The posterior probability that the woman is a carrier after the mutation is detected in the mother of the affected nephew is calculated as $1 / 21$ (Table 10). This is a little more than half of the $1 / 11$ posterior probability when genetic testing is not performed on the mother of the affected nephew.

Suppose an unaffected family member, who is more distant from the affected nephew (i.e., who is not between the woman and the affected nephew), is found to have one detectable mutation. There is a possibility that the detected mutation is not

Table 8

Bayesian analysis for a pregnant woman with an affected nephew who has been found to have only one detectable mutation A (Scenario 5)

\begin{tabular}{lccc}
\hline Pregnant woman & Carrier with Mutation A & Carrier with Mutation B & 0.25 \\
\hline Prior probability & 0.25 & 1 & 0.5 \\
Conditional probability of negative carrier screening & 0 & 0.25 & 1 \\
Joint probability & 0 & 0.5 & $1 / 3$ \\
Posterior probability & 0 & $2 / 3$ \\
\hline
\end{tabular}


Table 9

Bayesian analysis for the fetus of a pregnant woman with an affected nephew who has been found to have only one detectable mutation $\mathrm{A}^{a}$ (Scenario 5 )

\begin{tabular}{|c|c|c|c|c|c|c|c|c|c|}
\hline Pregnant woman & \multicolumn{6}{|c|}{ Carrier (Dm) } & \multicolumn{3}{|c|}{ Noncarrier } \\
\hline Prior probability & \multicolumn{6}{|c|}{$1 / 3$} & \multicolumn{3}{|c|}{$2 / 3$} \\
\hline \multirow[t]{2}{*}{ Father } & \multicolumn{4}{|c|}{ Carrier (Dp) } & \multicolumn{2}{|c|}{ Noncarrier } & \multicolumn{2}{|c|}{ Carrier } & Noncarrier \\
\hline & \multicolumn{4}{|c|}{0.04} & \multicolumn{2}{|c|}{0.96} & \multicolumn{2}{|c|}{0.04} & 0.96 \\
\hline \multirow[t]{2}{*}{ Fetus } & $\begin{array}{c}\text { Affected } \\
(\mathrm{Dp}+\mathrm{Dm})\end{array}$ & $\begin{array}{l}\text { Carrier } \\
(\mathrm{Dp})\end{array}$ & $\begin{array}{l}\text { Carrier } \\
(\mathrm{Dm})\end{array}$ & Noncarrier & $\begin{array}{l}\text { Carrier } \\
(\mathrm{Dm})\end{array}$ & Noncarrier & $\begin{array}{l}\text { Carrier } \\
(\mathrm{Dp})\end{array}$ & Noncarrier & Noncarrier \\
\hline & 0.25 & 0.25 & 0.25 & 0.25 & 0.5 & 0.5 & 0.5 & 0.5 & 1 \\
\hline $\begin{array}{l}\text { Negative prenatal } \\
\text { testing }\end{array}$ & $0.1 \times 1$ & 0.1 & 1 & 1 & 1 & 1 & 0.1 & 1 & 1 \\
\hline Joint probability & $1 / 3000$ & $1 / 3000$ & $10 / 3000$ & $10 / 3000$ & $480 / 3000$ & $480 / 3000$ & $4 / 3000$ & $40 / 3000$ & $1920 / 3000$ \\
\hline $\begin{array}{l}\text { Posterior } \\
\text { probability }\end{array}$ & $1 / 2946$ & $1 / 2946$ & $10 / 2946$ & $10 / 2946$ & $480 / 2946$ & $480 / 2946$ & $4 / 2946$ & $40 / 2946$ & $1920 / 2946$ \\
\hline
\end{tabular}

${ }^{a}$ The fetus tested negative in prenatal testing. Dp and Dm indicate paternally and maternally derived disease alleles. Because the pregnant woman tested negative in carrier screening, Dm, if present, has an undetectable mutation.

Table 10

Bayesian analysis for a pregnant woman with an affected nephew ${ }^{a}$ (Scenario 6)

\begin{tabular}{lcc}
\hline Pregnant woman & $\begin{array}{c}\text { Carrier with Mutation A present } \\
\text { in the affected nephew }\end{array}$ & $\begin{array}{c}\text { Carrier with Mutation B present } \\
\text { in the affected nephew }\end{array}$ \\
\hline $\begin{array}{l}\text { Prior probability } \\
\begin{array}{l}\text { Conditional probability that Mutation A is } \\
\text { detected in the mother of the affected nephew }\end{array}\end{array}$ & 0.25 & 0.25 \\
$\begin{array}{l}\text { Conditional probability that the woman is } \\
\text { negative in carrier screening }\end{array}$ & 0 & 0.5 \\
$\begin{array}{l}\text { Joint probability } \\
\text { Posterior probability }\end{array}$ & 0 & 0.1 \\
\hline
\end{tabular}

${ }^{a}$ The mother of the affected nephew has detectable Mutation A, and the woman is negative in carrier screening. Mutation A and Mutation B can be identical-by-state (IBS).

actually present in the affected nephew, especially if the disease allele frequency is relatively high in the general population. After the mutation is detected in the family member, the posterior probability that the woman is a carrier decreases by less than half, with the extent of the decrease depending on the distance between the family member with the detected mutation and the affected nephew.

\section{Scenario 7: Complex scenarios: Consultand negative by carrier testing, and proband or an unaffected family member with one detectable mutation using a different mutation panel}

Suppose a pregnant woman tests negative for mutations in $C F T R$, and that she has an affected nephew with only one detectable mutation, e.g., $\Delta$ F508 (F508del), but the nephew was tested using a different CFTR mutation panel. What is the probability that the woman is a carrier (Fig. 1)? For example, suppose the woman was tested using the ACMG, 25-mutation panel, and the nephew for $\Delta \mathrm{F} 508$ only. The prior probability that the woman is a carrier $(1 / 2)$ comprises a $1 / 4$ probability of carrying the $\Delta \mathrm{F} 508$ mutation detected in the nephew, and a $1 / 4$ probability of carrying a mutation undetectable by testing for $\Delta \mathrm{F} 508$ alone. The conditional probability that the woman tests negative if she carries $\Delta \mathrm{F} 508$, or if she is a noncarrier, is 0 or 1 , respectively. The conditional probability that the woman tests negative if she carries a mutation undetectable by testing for $\Delta$ F508 alone is $(1-0.9) /(1-0.7)$, i.e., the false-negative rate ( 1 - mutation detection rate) of the 25 -mutation panel divided by the false-negative rate ( 1 - mutation detection rate) of testing for $\Delta$ F508 alone (Fig. 1). In general, the conditional probability that the woman in this case tests negative if she carries a mutation undetectable by testing in the proband is $(1-[\mathrm{mu}-$ tation detection rate in the mother] / [1 - [mutation detection rate in the proband])]. The Bayesian analysis for this example is shown in Table 11. The posterior probability that the woman is a carrier is $1 / 7$.

Suppose the affected nephew is tested using the ACMG, 25mutation panel, which detects $90 \%$ of mutations in the nonHispanic Caucasian population and $\Delta$ F508 is detected but his second mutation is not. Suppose that the woman tests negative using an extended mutation panel that detects $93 \%$ of mutations in the non-Hispanic Caucasian population (Fig. 2). What is the probability that the woman is a carrier? The Bayesian 


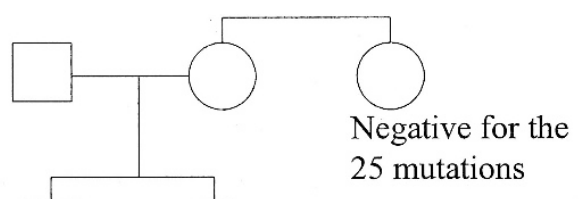

Tested only for $\Delta \mathrm{F} 508$

Carrier risk $1 / 2 \rightarrow ?$ Heterozygous $\triangle \mathrm{F} 508$

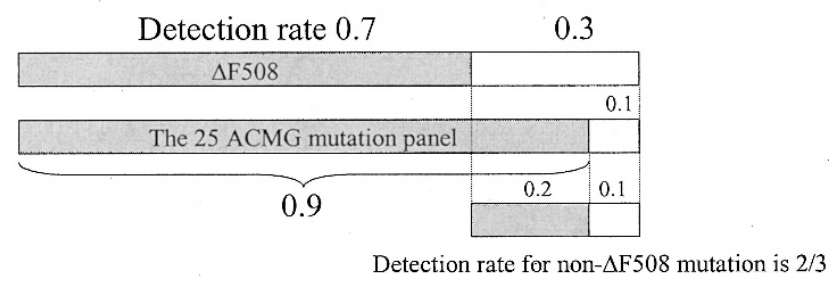

Fig. 1. Carrier screening for the 25 mutation panel on the mother who has an affected nephew with only one $\Delta$ F508 by test that detects only the $\Delta$ F508 mutation (Scenario 7).

analysis for this case is shown in Table 12. The conditional probability that the woman tests negative if she carries a mutation undetectable by the ACMG, 25-mutation panel is (1 $0.93) /(1-0.9)$. The posterior probability that the woman is a carrier is 0.26 .

\section{Scenario 8: Different mutation panel used in prenatal CFTR testing from the standard panel used in carrier screening of the parents}

This scenario will likely arise as technology advances and as testing more mutations than the current standard mutation panel becomes cost-effective. The Bayesian methods we described previously ${ }^{5}$ take into account only scenarios in which genetic testing for both the parents and the fetus is performed using the same mutation panel, and scenarios in which neither parent is tested. We illustrate herein methods for calculating genetic risks when different mutation panels are used for the parents and for the fetus. Hence, the Bayesian analyses for this scenario are modified from those used in our previous report. ${ }^{5}$ Each case assumes that both parents are non-Hispanic Caucasian, that the extended panel detects $93 \%$ of all mutations present in non-Hispanic Caucasians, and that the standard

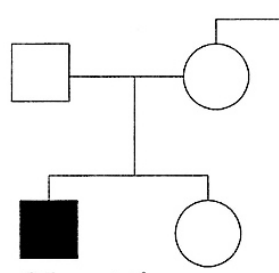

Tested for the 25 mutation

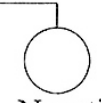

Negative for the extended panel that detects $93 \%$ of Caucasian mutations

Carrier risk $1 / 2 \rightarrow$ ? Heterozygous $\triangle \mathrm{F} 508$

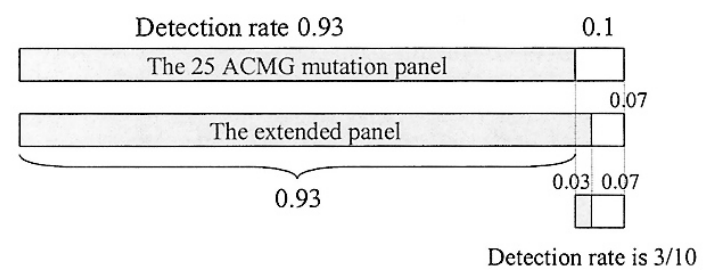

Fig. 2. Carrier screening for an extended mutation panel on the mother who has an affected nephew with only one $\Delta$ F508 by test that detects the ACMG 25 mutation panel (Scenario 7).

panel detects the 25 ACMG-recommended mutations, which comprise $90 \%$ of all mutations present in non-Hispanic Caucasians.

In Scenario 2 (Table 5) in Ogino et al., ${ }^{5}$ one of the parents (Parent A) tests negative, and the other parent (Parent B) has not been tested. The fetus has one detectable mutation by the extended panel. How does this information affect the risk calculations? The probability that a mutation in Parent A (Da), undetected by the standard panel, would be detectable by the extended panel is $0.3[=(0.93-0.9) /(1-0.9)]$. Therefore, the conditional probability of "No other mutation detected" in Column A would be $0.7[=(1-0.93) /(1-0.9)]$, instead of 1. The conditional probabilities of "One mutation identified in Da" in columns B and E are both $0.3[=(0.93-0.9) /(1-$ $0.9)$ ], instead of 0 . The Bayesian table is otherwise unchanged.

In the Scenario 3 (Table 6) in Ogino et al., ${ }^{5}$ one of the parents (Parent A) has a mutation detected by the standard panel and the other parent (Parent B) has not been tested. The fetus has one detectable mutation, which is the same mutation detected in Parent A. The conditional probability of "Allele from parent B" in Column A is now $1-0.93$, replacing " $1-d$." The Bayesian table is otherwise unchanged.

Table 11

Bayesian analysis for a woman with an affected nephew ${ }^{a}$ (Scenario 7)

\begin{tabular}{|c|c|c|c|}
\hline Pregnant woman & $\begin{array}{l}\text { Carrier with } \Delta F 508 \text { present } \\
\text { in the affected nephew }\end{array}$ & $\begin{array}{l}\text { Carrier with non- } \Delta \text { F508 mutation } \\
\text { present in the affected nephew }\end{array}$ & Noncarrier \\
\hline Prior probability & 0.25 & 0.25 & 0.5 \\
\hline $\begin{array}{l}\text { Conditional probability that the woman tests } \\
\text { negative }\end{array}$ & 0 & $(1-0.9) /(1-0.7)=1 / 3$ & 1 \\
\hline Joint probability & 0 & $1 / 12$ & $1 / 2$ \\
\hline Posterior probability & 0 & $1 / 7$ & $6 / 7$ \\
\hline
\end{tabular}

${ }^{a}$ The affected nephew has been shown to have only one $\Delta \mathrm{F} 508$ mutation by test that detects only the $\Delta \mathrm{F} 508$ mutation. The woman tested negative in carrier screening for the 25 mutations recommended by ACMG. 
Table 12

Bayesian analysis for a woman with an affected nephew ${ }^{a}$ (Scenario 7)

\begin{tabular}{lcc}
\hline Pregnant woman & $\begin{array}{c}\text { Carrier with } \Delta \text { F508 present } \\
\text { in the affected nephew }\end{array}$ & $\begin{array}{c}\text { Carrier with undetectable mutation } \\
\text { present in the affected nephew }\end{array}$ \\
\hline Prior probability & 0.25 & 0.25 \\
Conditional probability that the woman tests negative & 0 & $(1-0.93) /(1-0.9)=0.7$ \\
Joint probability & 0 & 0.175 \\
Posterior probability & 0 & 0.26 \\
\hline
\end{tabular}

${ }^{a}$ The affected nephew has been shown to have only one $\Delta \mathrm{F} 508$ mutation by test that detects $90 \%$ of mutations in the non-Hispanic Caucasian population. The woman tested negative in carrier screening for an extended mutation panel that comprises $93 \%$ of mutations in non-Hispanic Caucasian population.

In Scenario 4 (Table 7) in Ogino et al.,5 one of the parents (Parent A) has a mutation detected by the standard panel and other parent (Parent B) tests negative. The fetus has one detectable mutation, which is the same mutation detected in Parent A. The conditional probability of "Allele from parent B" in Column A is now $(1-0.93) /(1-0.9)=0.7$, instead of 1 . The conditional probability of "Allele from parent B" in Column C is now $0.3[=(0.93-0.9) /(1-0.9)]$, instead of 0 . The Bayesian table is otherwise unchanged.

In Scenario 6 (Table 9) in Ogino et al., ${ }^{5}$ one of the parents (Parent A) tests negative using the standard panel and the other parent (Parent B) has not been tested. The fetus tests negative using the extended panel. The conditional probabilities of "No mutation detected" in this case are shown in Table 13. Changes in the conditional probabilities are in bold face. The Bayesian table is otherwise unchanged.
In Scenario 7 (Table 10) in Ogino et al., ${ }^{5}$ both of the parents (Parent A and Parent B) test negative using the standard panel. The fetus tests negative using the extended panel. The conditional probabilities of "No mutation detected" in this case are shown in Table 14. Changes in the conditional probabilities are in bold face. The Bayesian table is otherwise unchanged.

In Scenario 8 (Table 11) in Ogino et al., ${ }^{5}$ one of the parents (Parent A) has a mutation detected by the standard panel and the other parent (Parent B) has not been tested. The fetus tests negative using the extended panel. The conditional probability of "The other allele with no mutation detected" in Column A is now $1-0.93$, replacing " $1-d$," and the conditional probability of "One allele with no mutation detected" in Column C is now $1-0.93$, replacing " $1-d$." The Bayesian table is otherwise unchanged.

Table 13

Generalized bayesian analysis for CF with one risk factor and no identifiable mutation ${ }^{a}$ (Scenario 8) (Modified from Table 9 in Ogino et al ${ }^{5}$ )

\begin{tabular}{|c|c|c|c|c|c|c|c|c|c|}
\hline Parent A & \multicolumn{6}{|c|}{ Carrier } & \multicolumn{3}{|c|}{ Noncarrier } \\
\hline Prior probability & \multicolumn{6}{|c|}{$x$} & \multicolumn{3}{|c|}{$1-x$} \\
\hline Test negative & \multicolumn{6}{|c|}{$1-c$} & \multicolumn{3}{|c|}{1} \\
\hline Parent B & \multicolumn{4}{|c|}{ Carrier } & \multicolumn{2}{|c|}{ Noncarrier } & \multicolumn{2}{|c|}{ Carrier } & Noncarrier \\
\hline Prior probability & \multicolumn{4}{|c|}{$y$} & \multicolumn{2}{|c|}{$1-y$} & \multicolumn{2}{|c|}{$y$} & $1-y$ \\
\hline \multirow[t]{2}{*}{ Fetus (mutation) } & $\begin{array}{c}\text { Affected } \\
(\mathrm{Da}+\mathrm{Db})\end{array}$ & $\begin{array}{l}\text { Carrier } \\
(\mathrm{Da})\end{array}$ & Carrier (Db) & Noncarrier & $\begin{array}{l}\text { Carrier } \\
(\mathrm{Da})\end{array}$ & Noncarrier & $\begin{array}{l}\text { Carrier } \\
(\mathrm{Db})\end{array}$ & Noncarrier & Noncarrier \\
\hline & 0.25 & 0.25 & 0.25 & 0.25 & 0.5 & 0.5 & 0.5 & 0.5 & 1 \\
\hline $\begin{array}{l}\text { Risk factor \#1 } \\
\text { (e.g., echogenic } \\
\text { bowel) }\end{array}$ & $a$ & $b$ & $b$ & $b^{\prime}$ & $b$ & $b^{\prime}$ & $b$ & $b^{\prime}$ & $b^{\prime}$ \\
\hline $\begin{array}{l}\text { No mutation } \\
\text { detected }\end{array}$ & $\begin{array}{c}(1-0.93) \\
{[(1-0.93) /} \\
(1-0.9)]\end{array}$ & $\begin{array}{l}(1-0.93) / \\
(1-0.9)\end{array}$ & $1-0.93$ & 1 & $\begin{array}{c}(1-0.93) / \\
(1-0.9)\end{array}$ & 1 & $1-0.93$ & 1 & 1 \\
\hline Joint probability & $\begin{array}{c}0.25 a x y \\
(1-0.93)^{2}\end{array}$ & $\begin{array}{c}0.25 b x y \\
(1-0.93)\end{array}$ & $\begin{array}{l}0.25 b x y \\
(1-c) \\
(1-0.93)\end{array}$ & $\begin{array}{c}0.25 b^{\prime} x y \\
(1-c)\end{array}$ & $\begin{array}{c}0.5 b x \\
(1-y) \\
(1-0.93)\end{array}$ & $\begin{array}{l}0.5 b^{\prime} x \\
(1-y) \\
(1-c)\end{array}$ & $\begin{array}{c}0.5 b y \\
(1-x) \\
(1-0.93)\end{array}$ & $\begin{array}{l}0.5 b^{\prime} y \\
(1-x)\end{array}$ & $\begin{array}{l}b^{\prime}(1-x) \\
\quad(1-y)\end{array}$ \\
\hline Column & A & B & C & $\mathrm{D}$ & E & $\mathrm{F}$ & $\mathrm{G}$ & $\mathrm{H}$ & I \\
\hline
\end{tabular}

${ }^{a}$ No detectable mutation has been found either in the fetus (for the extended panel), or in one of the parents (Parent A) (for the standard panel). The other parent (Parent B) has not been tested.

$a$, probability that fetus is positive for risk factor \#1 if fetus is affected; $b$, probability that fetus is positive for risk factor \# 1 if fetus is a carrier; $b^{\prime}$, probability that fetus is positive for risk factor \#1 if fetus is a noncarrier; $c$, mutation detection rate of genetic test for the ethnic background of parent A; Da, disease allele derived from parent $\mathrm{A} ; \mathrm{Db}$, disease allele derived from parent $\mathrm{B} ; x$, prior carrier probability for parent $\mathrm{A} ; y$, prior carrier probability for parent $\mathrm{B}$. 
Table 14

Generalized bayesian analysis for CF with one risk factor and no identifiable mutation ${ }^{a}$ (Scenario 8) (Modified from Table 10 in Ogino et al ${ }^{5}$ )

\begin{tabular}{|c|c|c|c|c|c|c|c|c|c|}
\hline Parent A & \multicolumn{6}{|c|}{ Carrier } & \multicolumn{3}{|c|}{ Noncarrier } \\
\hline Prior probability & \multicolumn{6}{|c|}{$x$} & \multicolumn{3}{|c|}{$1-x$} \\
\hline Test negative & \multicolumn{6}{|c|}{$1-c$} & \multicolumn{3}{|c|}{1} \\
\hline Parent B & \multicolumn{4}{|c|}{ Carrier } & \multicolumn{2}{|c|}{ Noncarrier } & \multicolumn{2}{|c|}{ Carrier } & Noncarrier \\
\hline Prior probability & \multicolumn{4}{|c|}{$y$} & \multicolumn{2}{|c|}{$1-y$} & \multicolumn{2}{|c|}{$y$} & $1-y$ \\
\hline Test negative & \multicolumn{4}{|c|}{$1-d$} & \multicolumn{2}{|c|}{1} & \multicolumn{2}{|c|}{$1-d$} & 1 \\
\hline \multirow[t]{2}{*}{$\begin{array}{l}\text { Fetus } \\
\quad \text { (mutation) }\end{array}$} & $\begin{array}{c}\text { Affected } \\
(\mathrm{Da}+\mathrm{Db})\end{array}$ & $\begin{array}{l}\text { Carrier } \\
(\mathrm{Da})\end{array}$ & $\begin{array}{l}\text { Carrier } \\
(\mathrm{Db})\end{array}$ & Noncarrier & $\begin{array}{l}\text { Carrier } \\
(\mathrm{Da})\end{array}$ & Noncarrier & $\begin{array}{l}\text { Carrier } \\
(\mathrm{Db})\end{array}$ & Noncarrier & Noncarrier \\
\hline & 0.25 & 0.25 & 0.25 & 0.25 & 0.5 & 0.5 & 0.5 & 0.5 & 1 \\
\hline $\begin{array}{l}\text { Risk factor \#1 } \\
\text { (e.g., } \\
\text { echogenic } \\
\text { bowel) }\end{array}$ & $a$ & $b$ & $b$ & $b^{\prime}$ & $b$ & $b^{\prime}$ & $b$ & $b^{\prime}$ & $b^{\prime}$ \\
\hline $\begin{array}{l}\text { No mutation } \\
\text { detected }\end{array}$ & $\begin{array}{c}{[(1-0.93) /} \\
(1-0.9)] \\
{[(1-0.93) /} \\
(1-0.9)]\end{array}$ & $\begin{array}{c}(1-0.93) / \\
(1-0.9)\end{array}$ & $\begin{array}{l}(1-0.93) / \\
(1-0.9)\end{array}$ & 1 & $\begin{array}{c}(1-0.93) / \\
(1-0.9)\end{array}$ & 1 & $\begin{array}{c}(1-0.93) / \\
(1-0.9)\end{array}$ & 1 & 1 \\
\hline Joint probability & $\begin{array}{c}0.25 a x y \\
(1-0.93)^{2}\end{array}$ & $\begin{array}{c}0.25 b x y \\
(1-d) \\
(1-0.93)\end{array}$ & $\begin{array}{c}0.25 b x y \\
(1-c) \\
(1-0.93)\end{array}$ & $\begin{array}{c}0.25 b^{\prime} x y \\
(1-c) \\
(1-d)\end{array}$ & $\begin{array}{c}0.5 b x \\
(1-y) \\
(1-0.93)\end{array}$ & $\begin{array}{l}0.5 b^{\prime} x \\
(1-y) \\
(1-c)\end{array}$ & $\begin{array}{c}0.5 b y \\
(1-x) \\
(1-0.93)\end{array}$ & $\begin{array}{l}0.5 b^{\prime} y \\
(1-x) \\
(1-d)\end{array}$ & $\begin{array}{c}b^{\prime}(1-x) \\
(1-y)\end{array}$ \\
\hline Column & A & B & $\mathrm{C}$ & $\mathrm{D}$ & $\mathrm{E}$ & $\mathrm{F}$ & $\mathrm{G}$ & $\mathrm{H}$ & I \\
\hline
\end{tabular}

${ }^{a}$ No detectable mutation has been found in the fetus (for the extended panel), or in either of the parents (for the standard panel).

$a$, probability that fetus is positive for risk factor \# 1 if fetus is affected; $b$, probability that fetus is positive for risk factor \#1 if fetus is a carrier; $b^{\prime}$, probability that fetus is positive for risk factor \#1 if fetus is a noncarrier; $c$, mutation detection rate of genetic test for the ethnic background of parent A; d, mutation detection rate of genetic test for the ethnic background of parent $\mathrm{B}$; Da, disease allele derived from parent $\mathrm{A}$; $\mathrm{Db}$, disease allele derived from parent $\mathrm{B} ; x$, prior carrier probability for parent $\mathrm{A} ; y$, prior carrier probability for parent $\mathrm{B}$.

In Scenario 9 (Table 12) in Ogino et al., 5 one of the parents (Parent A) has a mutation detected by the standard panel and the other parent (Parent B) tests negative. The fetus tests negative using the extended panel. The conditional probability of "The other allele with no mutation detected" in Column A is now $(1-0.93) /(1-0.9)$, replacing " 1 ," and the conditional probability of "One allele with no mutation detected" in Column C is now $(1-0.93) /(1-0.9)$, replacing "1." The Bayesian table is otherwise unchanged.

\section{Scenario 9: Carrier screening for an individual with mixed ethnicity}

For individuals with mixed ethnicity, genetic risk assessments are often quite complex. Suppose a woman with a nonHispanic Caucasian father, a Hispanic mother and no family history of $\mathrm{CF}$ undergoes carrier screening. If she tests negative using the ACMG, 25-mutation panel, what is the probability that she is a carrier? The Bayesian analysis is shown in Table 15. We assume that the carrier frequencies for CF in non-Hispanic Caucasian and Hispanic populations are $1 / 25$ and $1 / 46$, respectively, and that the ACMG panel detects $90 \%$ and $57 \%$ of mutations, respectively. The probability that the individual is a carrier is the sum of the posterior probabilities in Columns A, $\mathrm{B}, \mathrm{D}$, and $\mathrm{F}$, or 0.0067 .

\section{DISCUSSION}

Bayesian analysis plays an essential role in calculations of genetic risk. ${ }^{1-3,10}$ We previously developed generalizable Bayesian methods to calculate autosomal recessive disease risk when only one or no mutation is detected and multiple mutations have been identified in the disease gene. ${ }^{5}$ These methods are particularly useful for CF. Prenatal screening for CF presents a number of complex challenges, including different methods for mutation detection, laboratory quality assurance issues, education, reporting, residual risk calculations, and genetic counseling. ${ }^{11}$ Using concrete examples, we have illustrated herein principles of genetic risk calculations for a variety of common scenarios, for which our previous methods are inadequate. For example, as the technology of mutation detection advances, more mutations will be screened at lower costs, and mutation panels such as those for CF will likely include many more mutations. We have provided methods for genetic risk calculations for scenarios in which the proband or another family member has been tested using a different mutation panel from that used for the consultand (see Scenario 7), and in which the fetus is tested by a mutation panel different from that used for screening of the parents (see Scenario 8). The ACMG-recommended CF mutation panel will soon be reduced to 23 mutations, but this will not alter any of our calculations significantly. 
Table 15

Bayesian analysis for an individual with mixed ethnicity and negative carrier screening (Scenario 9)

\begin{tabular}{|c|c|c|c|c|c|c|c|c|}
\hline Father & \multicolumn{5}{|c|}{ Carrier } & \multicolumn{3}{|c|}{ Noncarrier } \\
\hline $\begin{array}{l}\text { Prior } \\
\text { probability }\end{array}$ & \multicolumn{5}{|c|}{$1 / 25$} & \multicolumn{3}{|c|}{$24 / 25$} \\
\hline Mother & & Carrier & & \multicolumn{2}{|c|}{ Noncarrier } & \multicolumn{2}{|c|}{ Carrier } & Noncarrier \\
\hline $\begin{array}{l}\text { Prior } \\
\text { probability }\end{array}$ & & $1 / 46$ & & \multicolumn{2}{|c|}{$45 / 46$} & \multicolumn{2}{|c|}{$1 / 46$} & $45 / 46$ \\
\hline \multirow[t]{2}{*}{ Consultand } & $\begin{array}{l}\text { Carrier } \\
(\mathrm{Dp})\end{array}$ & $\begin{array}{l}\text { Carrier } \\
(\mathrm{Dm})\end{array}$ & Noncarrier & $\begin{array}{l}\text { Carrier } \\
(\mathrm{Dp})\end{array}$ & Noncarrier & $\begin{array}{l}\text { Carrier } \\
(\mathrm{Dm})\end{array}$ & Noncarrier & Noncarrier \\
\hline & $1 / 4$ & $1 / 4$ & $1 / 4$ & $1 / 2$ & $1 / 2$ & $1 / 2$ & $1 / 2$ & 1 \\
\hline $\begin{array}{l}\text { Conditional } \\
\text { probability } \\
\text { of negative } \\
\text { screening }\end{array}$ & $1-0.9$ & $1-0.57$ & 1 & $1-0.9$ & 1 & $1-0.57$ & 1 & 1 \\
\hline $\begin{array}{l}\text { Joint } \\
\text { probability }\end{array}$ & $10 / 460000$ & $43 / 460000$ & $100 / 460000$ & $900 / 460000$ & $9000 / 460000$ & $2064 / 460000$ & $4800 / 460000$ & $432000 / 460000$ \\
\hline $\begin{array}{l}\text { Posterior } \\
\text { probability }\end{array}$ & 0.000022 & 0.000096 & 0.00022 & 0.0020 & 0.020 & 0.0046 & 0.011 & 0.96 \\
\hline Column & A & B & $\mathrm{C}$ & $\mathrm{D}$ & E & $\mathrm{F}$ & G & $\mathrm{H}$ \\
\hline
\end{tabular}

Dp, disease allele from the father; $\mathrm{Dm}$, disease allele from the mother.

Prenatal and carrier screening strategy is still a controversial topic. ${ }^{11,12}$ To decrease complexity in genetic counseling, a couple screening strategy was proposed. ${ }^{13}$ From a risk calculation point of view, however, couple screening may actually increase the uncertainty of genetic risk assessments when a couple comprises two individuals with different ethnic backgrounds. Further complications arise when only one member of a couple tests positive and a relative of either member presents for genetic counseling.

Another controversial topic is neonatal screening for CF. We have developed methods to calculate $\mathrm{CF}$ risks in various neonatal screening scenarios (Ogino et al., unpublished data, 2004).

It is difficult to provide a hard and fast rule for how high the CF risk for a given fetus should be before considering or recommending further testing. The decision depends on a number of criteria, including the willingness of the parents to pay additional costs, the degree of the parents' anxiety, and the improvement in the accuracy of risk calculations provided by additional testing. Keeping these criteria in mind, decisions regarding additional testing need to be made on a case-by-case basis.

Although echogenic bowel is usually detected rather late in pregnancy, proper counseling and testing should include both parents. The accuracy of risk assessments increases as more information becomes available, and risk assessments are most accurate when both parents (and the proband, if applicable), in addition to the fetus, are tested. Note that in testing and risk assessment for $\mathrm{CF}$, the conditional probabilities of echogenic bowel should be updated when data on ethnicity-specific frequencies of echogenic bowel among affected fetuses, carrier fetuses, and noncarrier fetuses become available. With regard to frequencies of echogenic bowel, our calculations currently depend on the single large study by Scotet et al. ${ }^{6}$ In the future, more population data should become available, increasing the accuracy of genetic risk assessments.

In conclusion, we present a variety of versatile Bayesian methods. They are applicable to many common clinical scenarios and allow autosomal recessive disease probabilities and carrier probabilities to be calculated accurately, taking into account all relevant information. Our methods allow accurate genetic risk estimates for patients and their family members, for $\mathrm{CF}$ or other autosomal recessive disorders.

\section{Acknowledgments}

The content of this publication does not necessarily reflect the views or policies of the Department of Health and Human Services, nor does mention of trade names, commercial products, or organizations imply endorsement by the U.S. Government. This project has been funded in whole or in part with Federal Funds from the National Cancer Institute, National Institutes of Health.

\section{References}

1. Young ID. Introduction to risk calculation in genetic counseling, 2nd ed. Oxford: Oxford University Press, 1999.

2. Bridge PJ. The calculation of genetic risks: Worked examples in DNA diagnostics, Second Edition ed. Baltimore: The Johns Hopkins University Press, 1997.

3. Ogino S, Wilson RB. Bayesian analysis and risk assessment in genetic counseling and testing. J Mol Diagn 2004;6:1-9.

4. Hodge SE, Flodman PL. Risk calculations: Still essential in the molecular age. Am J Med Genet In press.

5. Ogino S, Wilson RB, Grody WW. Bayesian risk assessment for autosomal recessive diseases: Fetal echogenic bowel with one or no detectable CFTR mutation. J Med Genet 2004;41:e70.

6. Scotet V, De Braekeleer M, Audrezet MP, Quere I, Mercier B, Dugueperoux I et al. Prenatal detection of cystic fibrosis by ultrasonography: A retrospective study of more than 346000 pregnancies. J Med Genet 2002;39:443-448. 
7. Richards CS, Bradley LA, Amos J, Allitto B, Grody WW, Maddalena A et a. Standards and guidelines for CFTR mutation testing. Genet Med 2002;4:379-391.

8. Grody WW, Cutting GR, Klinger KW, Richards CS, Watson MS, Desnick RJ. Laboratory standards and guidelines for population-based cystic fibrosis carrier screening. Genet Med 2001;3:149-154.

9. Bobadilla JL, Macek M Jr, Fine JP, Farrell PM. Cystic fibrosis: A worldwide analysis of CFTR mutations-correlation with incidence data and application to screening. Hum Mutat 2002;19:575-606.
10. Ogino S, Wilson RB. Genetic testing and risk assessment for spinal muscular atrophy (SMA). Hum Genet 2002;111:477-500.

11. Richards CS, Grody WW. Prenatal screening for cystic fibrosis: Past, present and future. Expert Rev Mol Diagn 2004;4:49-62.

12. Wald NJ, Morris JK, Rodeck CH, Haddow JE, Palomaki GE. Cystic fibrosis: Selecting the prenatal screening strategy of choice. Prenat Diagn 2003;23:474483.

13. Wald NJ. Couple screening for cystic fibrosis. Lancet 1991;338:1318-1319. 